\title{
Evidence of superfluidity in a dipolar supersolid from nonclassical rotational inertia
}

\author{
L. Tanzi ${ }^{1,2}$, J. G. Maloberti ${ }^{1,2}$, G. Biagioni ${ }^{1,2}$, A. Fioretti', C. Gabbanini', G. Modugno, ${ }^{1,2, *}$ \\ ${ }^{1}$ CNR-INO, Sede Secondaria di Pisa, 56124 Pisa, Italy. 2LENS and Dipartimento di Fisica e Astronomia, Università di Firenze, 50019 Sesto Fiorentino, Italy. \\ *Corresponding author. Email: modugno@lens.unifi.it
}

\begin{abstract}
A key manifestation of superfluidity in liquids and gases is a reduction of the moment of inertia under slow rotations. Non-classical rotational effects have also been considered in the context of the elusive supersolid phase of matter, in which superfluidity coexists with a lattice structure. Here we show that the recently discovered supersolid phase in dipolar quantum gases features a reduced moment of inertia. Using a dipolar gas of dysprosium atoms, we study a peculiar rotational oscillation mode in a harmonic potential, the scissors mode, previously investigated in ordinary superfluids. From the measured moment of inertia, we deduce a superfluid fraction that is different from zero and of order of unity, providing direct evidence of the superfluid nature of the dipolar supersolid.
\end{abstract}

Superfluids exhibit their most spectacular properties during rotation. This is because the superfluid state is described by a macroscopic wavefunction, whose phase can change only by integer multiples of $2 \pi$ upon completing a closed path. For a cylindrical superfluid rotating at low angular velocities, $\omega$ $\rightarrow 0$, this condition leads to the vanishing of both angular momentum $L$ and moment of inertia $I=\langle L\rangle / \omega$. An angular momentum can appear only for sufficiently large $\omega$ at integer multiples of the reduced Planck's constant $\hbar$, through the appearance of quantized vortices. These non-classical rotational effects have been verified for most known superfluids: nuclear matter (1), ${ }^{4} \mathrm{He}(2),{ }^{3} \mathrm{He}(3)$, gaseous Bose-Einstein condensates (4), degenerate Fermi gases (5), and excitonpolariton condensates (6). A related phenomenon is the Meissner effect in superconductors (7).

At the end of the '60, another type of bosonic phase of matter described by a macroscopic wavefunction, the supersolid, was predicted to exist. In a supersolid, superfluidity coexists with a crystal-type structure (8-10). A. J. Leggett suggested that a rotating supersolid should show a moment of inertia intermediate between those of a superfluid and of a classical system, $I=\left(1-f_{s}\right) I_{c}$. Here, $I_{c}$ is the classical moment of inertia and $0 \leq f_{s} \leq 1$ is the so-called superfluid fraction (10). This phenomenon is called non-classical rotational inertia (NCRI). Standard superfluids can have $f_{s}<1$, but only at finite temperature, $\mathrm{T}>0$, thanks to the presence of a thermal component. In a supersolid at $T=0$, the reduction of the superfluid fraction is instead caused by the spatially modulated density, which tends to increase the inertia toward the classical limit $(10,11)$.

At the time it was proposed, the primary candidate for observing supersolidity was solid helium. Torsion oscillators were employed extensively to attempt detecting NCRI (12).
The original announcement of the possible presence of a large superfluid fraction, $f_{s} \approx 10^{-1}(13,14)$, has later received a different interpretation based on a change of the elastic properties of the solid (15) and has not been confirmed by more recent studies (16). Superfluidity in bulk solid helium has now been excluded down to the level of $10^{-4}$ (17), and the search goes on in 2D films (18).

Here, we study a different supersolid candidate, a gaseous Bose-Einstein condensate (BEC) of strongly dipolar atoms, where a density-modulated regime coexisting with the phase coherence necessary for supersolidity has been recently discovered (19-21). So far, its superfluid nature has been tested through non-rotational excitation modes that can be described in terms of the hydrodynamic equations for superfluids (22-24). Here we aim instead at characterizing the NCRI of such system, searching for direct evidence of superfluidity under rotation, in the spirit of the helium experiments.

Achieving dipolar supersolids large enough to realize a cylindrical geometry is so far not possible, so we employ a specific rotation technique that fits the asymmetric, small-sized systems available in the laboratory. We excite the so-called scissors mode, a small-angle rotational oscillation of the harmonic potential that naturally holds the system. This technique, inspired by an excitation mode of nuclei (25), has been proposed $(26,27)$ and employed $(28)$ to demonstrate superfluidity of ordinary BECs. A recent theoretical study has shown that the scissors mode can also be used to characterize the NCRI of a dipolar supersolid (29). We study the change of the scissors mode frequency across the transition from BEC to the supersolid regime, so that we can directly compare the supersolid with a fully superfluid system.

In the experiment, a BEC of strongly magnetic Dy atoms is held in an anisotropic harmonic trap, with frequencies $\omega_{x, y, z}$ 
$=2 \pi(23,46,90) \mathrm{s}^{-1}$, with the dipoles oriented in the $z$ direction by a magnetic field $B$ (Fig. 1). The temperature is sufficiently low to have a negligible thermal component (30). We induce the transition from BEC to supersolid by tuning via a magnetic Feshbach resonance the interaction parameter $\varepsilon_{d d}$, which parametrizes the ratio of the dipolar and van der Waals interaction energies (19). In the supersolid regime, a density modulation develops along the weak $x$ axis, leading to the appearance of interference peaks in the momentum distribution. We expect our lattice to be composed of two principal density maxima, or "droplets", each containing about $10^{4}$ atoms (22). This realizes a so-called cluster supersolid (31), very different from the hypothesized helium supersolid with one particle per lattice site. In principle, further tuning of $\varepsilon_{d d}$ would bring the system into the so-called droplet crystal regime, with no coherence between the droplets (1921).

The scissors mode is excited by changing suddenly the direction of the eigenaxes of the harmonic trap (30). This results in a sinusoidal oscillation with frequency $\omega_{s c}$ of the angle $\theta$ between the long axis of the system and the corresponding trap axis. We choose to rotate the system in the $(x, y)$ plane, perpendicular to the direction of the dipoles, in order for the dipolar interaction potential to be independent of $\theta(32,33)$.

The oscillation frequency can be directly related to the moment of inertia of the superfluid through:

$$
I=I_{c} \alpha \beta\left(\omega_{x}^{2}+\omega_{y}^{2}\right) / \omega_{s c}^{2}(1)
$$

Where $\alpha=\left(\omega_{y}{ }^{2}-\omega_{x}{ }^{2}\right) /\left(\omega_{y}{ }^{2}+\omega_{x}{ }^{2}\right)$ and $\beta=\left\langle x^{2}-y^{2}\right\rangle /\left\langle x^{2}+y^{2}\right\rangle$ are geometrical factors measuring the deviation from cylindrical symmetry of the trap and of the density distribution, respectively $(26,29)$. Whereas $\alpha$ can be measured experimentally, $\beta$ needs to be calculated theoretically (30). For non-dipolar BECs in the Thomas-Fermi regime, one has the simplification $\beta=\alpha(27)$. For dipolar systems, the density deformation changes instead with the interaction parameter owing to magnetostriction, $\beta=\beta\left(\varepsilon_{d d}\right) \neq \alpha$ (32). If the oscillation amplitude is much smaller than $\beta$, the density deformation stays constant during the motion (26).

We can now connect the moment of inertia to a superfluid fraction, which we define specifically for our system in analogy with Leggett's definition, taking into account our non-cylindrical geometry:

$$
I=\left(1-f_{s}\right) I_{c}+f_{s} \beta^{2} I_{c \cdot}(2)
$$

It is easy to see that this definition coincides with Leggett's one in the cylindrical case, $\beta=0$. It also coincides with the known results for a superfluid with elliptical geometry, $I=\beta^{2}$ $I_{c}(1,26,34)$. The presence of a residual moment of inertia in the $\mathrm{BEC}$, despite $f_{s}=1$ at $\mathrm{T}=0$, derives from a peculiar velocity distribution, which is very different from the one in a cylindrical geometry $(26,27)$. Finally, by combining Eqs. 1 and 2 one can directly relate the superfluid fraction to the trap and scissors frequencies and to the deformation:

$$
f_{s}=\left[1-\alpha \beta\left(\omega_{x}{ }^{2}+\omega_{y}{ }^{2}\right) / \omega_{s c}{ }^{2}\right] /\left(1-\beta^{2}\right)(3)
$$

We note that the scissors mode is analogous to the helium torsion oscillators because both detect NCRI via the oscillation frequency (13-16), although there are some differences. In the scissors mode, all atoms experience the restoring force from the trap, so there are no elastic effects to consider (15). A finite deformation $\beta$ is clearly necessary for the scissors mode, whereas torsion oscillators are normally symmetric, although macroscopic deformations can be taken into account with the same formalism $(34,35)$ and a related tortuosity effect is present for superfluids in porous media (13).

Let us now turn to the experimental results. Figure 1, B to $\mathrm{E}$, summarize the scissors measurements in the BEC and supersolid regimes. The $2 \mathrm{D}$ density distributions are imaged after a free expansion of the system, representing effective momentum distributions. They are fitted to extract the angle $\theta$ ' in the laboratory frame for various observation times $t$. The resulting data for $\theta^{\prime}(t)$ are fitted with a sinusoid to measure $\omega_{s c}(30)$. Both BEC and supersolid regimes feature single-frequency oscillations, as expected for weakly-interacting superfluids (26). We have checked that a thermal sample features instead a two-frequency oscillation, as expected for a weakly interacting system, see Fig. S1 (30).

To avoid perturbations caused by other collective modes (30), we employ two different excitation techniques for the $\mathrm{BEC}$ and the supersolid regimes, which result in a lower amplitude of the scissors mode for the supersolid, see Fig. 1, D and E. The accuracy in the determination of the scissors frequency in that regime is limited also by the finite lifetime of the supersolid (19).

A summary of the experimental results for the scissors frequency and the related moment of inertia is shown in Fig. 2. The results are compared to the theoretical predictions of Ref. 29, calculated for trap parameters and atom numbers close to the experimental ones. For the BEC, we measure a frequency that depends only weakly on the interaction parameter $\varepsilon_{d d}$, consistent with the prediction of a weak change of the deformation $\beta\left(\varepsilon_{d d}\right)$ (32). In contrast, when the system enters the supersolid regime we observe a clear reduction of the frequency, in agreement with the theory. From the measured frequency, we can determine the moment of inertia $I / I_{c}$ through Eq. 1, where the deformation $\beta$ is determined from the numerically calculated density distributions (29). The results are shown in Fig. 2B. In the BEC regime, the moment of inertia differs by a factor of two from the classical value and the ratio $I / I_{c}$ is consistent with $\beta^{2}$, as expected for a fully 
superfluid system. In the supersolid regime, at $\varepsilon_{d d}=1.45$, the moment of inertia increases toward the classical value, but does not reach it. This provides evidence of NCRI for the dipolar supersolid.

The data point in Fig. 2B further in the supersolid regime, at $\varepsilon_{d d}=1.5$ has larger error bars owing to the shorter lifetime of the system. We were unable to study the droplet crystal regime, which is predicted to appear for $\varepsilon_{d d} \approx 1.52$ (29), because of the loss of the interference pattern (19-21).

The change of $I / I_{c}$ is in principle caused by both the change of shape, $\beta\left(\varepsilon_{d d}\right)$, when the supersolid modulation forms and the related change of the superfluid fraction. The experiment-theory agreement for $I / I_{c}$ both in the BEC regime, where $f_{s}=1$, and at $\varepsilon_{d d}=1.45$, where $I$ is expected to be close to $I_{c}$, supports the validity of the calculated $\beta$ for our system. Equation 2 shows that if the superfluid fraction of the supersolid varies between 0 and 1 , then $I / I_{c}$ shown in Fig. $2 B$ should vary between 1 and $\beta^{2}$. More directly, we calculate the superfluid fraction from Eq. 3, employing the experimental frequencies and the theoretical $\beta$. The results are shown in Fig. 3 , together with the corresponding points calculated from the theoretical predictions of ref.29.

In the BEC regime, the data confirm that the system is fully superfluid, $f_{s}=1$, as already found for non-dipolar BECs (28). In the supersolid regime, we can reliably calculate the superfluid fraction only for the experimental data point at $\varepsilon_{d d}$ $=1.45$. Remarkably, the superfluid fraction of the supersolid is very large, $f_{s} \approx 0.9$, in agreement with the numerical calculations. Given the measurement uncertainty, $f_{s}$ is consistent with unity and inconsistent with zero. This result demonstrates the superfluid nature of the dipolar supersolid under rotation.

The theory predicts a reduction of the superfluid fraction moving further into the supersolid regime, although $f_{\mathrm{s}}$ remains finite even in the droplet crystal regime because of the superfluidity of the individual droplets under rotation (29). In the experiment, we cannot check whether $f_{s}$ decreases moving to $\varepsilon_{d d}=1.5$, because the lower measurement accuracy and the increase of $\beta^{2}$ shown in Fig. 2B prevent us from measuring $f_{s}$ reliably (30).

It is interesting to compare our results to the original prediction by Leggett for the superfluid fraction of a supersolid rotating in a one-dimensional annulus,

$$
f_{s} \leq\left(\int d x / \rho(x)\right)^{-1}(4)
$$

Where $\rho(x)$ is the normalized density along the annulus and the integral is performed on a lattice cell $(10,11)$. Equation 4 shows that the reduction of the superfluid fraction is a consequence of the breaking of translational invariance, as $f_{s}$ is determined by the minimum density between lattice sites. Intuitively, in a homogenous superfluid, $\rho(x)=$ const implies that each atom is equally delocalized so no rotation happens. In a system where $\rho(x) \rightarrow 0$ between neighboring lattice sites, the sites are distinguishable so the system rotates classically. The supersolid is the intermediate case in which the atoms are still delocalized but the density modulation allows a partial rotation, increasing the moment of inertia when compared to a homogeneous superfluid.

In 1970, Leggett used Eq. 4 and the known information on the helium lattice to estimate $f_{s}<10^{-4}$ for solid helium (10), a result compatible with current measurements (17). Our dipolar supersolid does not move in a $1 \mathrm{D}$ configuration as in the Leggett model but has a more complex dynamics in the whole $(x, y)$ plane, with both motion along the $x$ axis, where the density modulation forms, and rotation of the individual droplets. Therefore, we expect Eq. 4 to account only for the superfluid fraction related to the dynamics along $x$, as it does not consider the superfluidity of the individual droplets.

Because we cannot measure $\rho(x)$ experimentally, we employ numerical calculations (30). The right-hand side of Eq. 4 is shown in Fig. 3 as triangles. It drops from unity for the BEC to about 0.3 for the supersolid, a relatively large value set by the large overlap between the two central droplets (see Fig. 3, inset). It then decreases for increasing $\varepsilon_{d d}$, reaching almost zero at $\varepsilon_{d d}=1.5$, where the droplets overlap almost vanishes. In that regime, one can recover the finite superfluid fraction of the numerical calculations by considering the droplets' superfluidity. Indeed, applying Eq. 2 to the case of independent droplets and considering that each droplet's moment of inertia about its axis is zero thanks to the cylindrical symmetry $(30)$, one obtains the estimate $f_{s}^{d r o p} \approx(1-\beta) /(1$ $-\beta^{2}$ ). Using the theoretical distributions, we get $f_{s}^{\text {drop }} \approx 0.5$ for all the data points in the supersolid regime (black diamonds in Fig. 3). This estimate is quite close to the numerical data point for $f_{s}$ at $\varepsilon_{d d}=1.5$, and more than 2 standard deviations below the experimental data point at $\varepsilon_{d d}=1.45$. Together with the qualitatively similar reduction of the two theoretical datasets for increasing $\varepsilon_{d d}$, this suggests that the mechanism identified by Leggett might have a relevant role in our small dipolar supersolid. To obtain a quantitative assessment, one will need further measurements and a theoretical analysis based on a 2D analog of the Leggett result $(36,37)$.

We have established the superfluid nature of the dipolar supersolid by characterizing its non-classical rotational inertia. The supersolid is different from standard superfluids because of the reduced superfluid fraction thanks to the spontaneous breaking of translational invariance. The techniques we have demonstrated, with an improvement of the measurement precision and of the resolution on $\varepsilon_{d d}$, will allow testing whether the superfluid fraction of the supersolid is indeed smaller than unity. Achieving larger systems might also allow studying the supersolid behavior in an annular geometry or in a 2D configuration, as well as studying the dynamics of quantized vortices in the supersolid phase (29). 


\section{REFERENCES AND NOTES}

1. A. B. Migdal, Superfluidity and the moments of inertia of nuclei. Sov. Phys. JETP 10, 176-185 (1960); http://www.jetp.ac.ru/cgi-bin/dn/e_010_01_0176.pdf.

2. G. B. Hess, W. M. Fairbank, Measurements of the angular momentum in superfluid helium. Phys. Rev. Lett. 19, 216-218 (1967). doi:10.1103/PhysRevLett.19.216

3. P. J. Hakonen, O. T. Ikkala, S. T. Islander, O. V. Lounasmaa, T. K. Markkula, P. Roubeau, K. M. Saloheimo, G. E. Volovik, E. L. Andronikashvili, D. I. Garibashvili, J. S. Tsakadze, NMR experiments on rotating superfluid ${ }^{3} \mathrm{He}-\mathrm{A}$ : Evidence for vorticity. Phys. Rev. Lett. 48, 1838-1841 (1982). doi:10.1103/PhysRevLett.48.1838

4. F. Chevy, K. W. Madison, J. Dalibard, Measurement of the angular momentum of a rotating bose-einstein condensate. Phys. Rev. Lett. 85, 2223-2227 (2000). doi:10.1103/PhysRevLett.85.2223 Medline

5. M. W. Zwierlein, J. R. Abo-Shaeer, A. Schirotzek, C. H. Schunck, W. Ketterle, Vortices and superfluidity in a strongly interacting Fermi gas. Nature 435, 1047-1051 (2005). doi:10.1038/nature03858 Medline

6. K. G. Lagoudakis, M. Wouters, M. Richard, A. Baas, I. Carusotto, R. André, B. Le Si Dang, B. Deveaud-Plédran, Quantized vortices in an exciton-polariton condensate. Nat. Phys. 4, 706-710 (2008). doi:10.1038/nphys105]

7. A. J. Leggett, "Superconductivity and superfluidity: Basic phenomenology," in Quantum Liquids: Bose Condensation and Cooper Pairing in Condensed-Matter Systems, (Oxford Univ. Press, ed. 1, 2006), pp. 20-26.

8. A. F. Andreev, I. M. Lifshitz, Quantum theory of defects in crystals. Sov. Phys. JETP 29, 1107-1113 (1969); http://jetp.ac.ru/cgi-bin/dn/e_029 06_1107.pdf.

9. G. V. Chester, Speculations on Bose-Einstein condensation and quantum crystals. Phys. Rev. A 2, 256-258 (1970). do: 10.1103/PhysRevA.2.256

10. A. J. Leggett, Can a solid be "superfluid"? Phys. Rev. Lett. 25, 1543-1546 (1970). doi:10.1103/PhysRevLett.25.1543

11. A. J. Leggett, On the superfluid fraction of an arbitrary many-body system at $\mathrm{T}=0$. J. Stat. Phys. 93, 927-941 (1998). doi:10.1023/B:JOSS.0000033170.38619.6c

12. M. H. W. Chan, R. B. Hallock, L. Reatto, Overview on solid ${ }^{4} \mathrm{He}$ and the issue of supersolidity. J. Low Temp. Phys. 172, 317-363 (2013). doن:10.1007/s10909-013$0882-x$

13. E. Kim, M. H. W. Chan, Probable observation of a supersolid helium phase. Nature 427, 225-227 (2004). doi:10.1038/nature02220 Medline

14. E. Kim, M. H. W. Chan, Observation of superflow in solid helium. Science 305, 19411944 (2004). doi:10.1126/science.1101501 Medline

15. J. Day, J. Beamish, Low-temperature shear modulus changes in solid ${ }^{4} \mathrm{He}$ and connection to supersolidity. Nature 450, 853-856 (2007). doi:10.1038/nature06383 Medline

16. D. Y. Kim, M. H. W. Chan, Absence of supersolidity in solid helium in porous Vycor glass. Phys. Rev. Lett. 109, 155301 (2012). doi:10.1103/PhysRevLett.109.155301 Medline

17. A. Eyal, X. Mi, A. V. Talanov, J. D. Reppy, Search for supersolidity in solid ${ }^{4} \mathrm{He}$ using multiple-mode torsional oscillators. Proc. Natl. Acad. Sci. U.S.A. 113, E3203E3212 (2016). doi:10.1073/pnas.1605738113 Medline

18. J. Nyéki, A. Phillis, A. Ho, D. Lee, P. Coleman, J. Parpia, B. Cowan, J. Saunders, Intertwined superfluid and density wave order in two-dimensional ${ }^{4} \mathrm{He}$. Nat. Phys. 13, 455-459 (2017). doi:10.1038/nnhys4023

19. L. Tanzi, E. Lucioni, F. Famà, J. Catani, A. Fioretti, C. Gabbanini, R. N. Bisset, L. Santos, G. Modugno, Observation of a dipolar quantum gas with metastable supersolid properties. Phys. Rev. Lett. 122, 130405 (2019) doi:10.1103/PhysRevLett.122.130405 Medline

20. F. Böttcher, J.-N. Schmidt, M. Wenzel, J. Hertkorn, M. Guo, T. Langen, T. Pfau, Transient supersolid properties in an array of dipolar quantum droplets. Phys. Rev. X9, 011051 (2019). doi:10.1103/PhysRevX.9.011051

21. L. Chomaz, D. Petter, P. Ilzhöfer, G. Natale, A. Trautmann, C. Politi, G. Durastante, R. M. W. van Bijnen, A. Patscheider, M. Sohmen, M. J. Mark, F. Ferlaino, M. J. Mark, F. Ferlaino, Long-lived and transient supersolid behaviors in dipolar quantum gases. Phys. Rev. X 9, 021012 (2019). doi:10.1103/PhysRevX.9.021012

22. L. Tanzi, S. M. Roccuzzo, E. Lucioni, F. Famà, A. Fioretti, C. Gabbanini, G. Modugno, A. Recati, S. Stringari, Supersolid symmetry breaking from compressional oscillations in a dipolar quantum gas. Nature 574, 382-385 (2019). doi:10.1038/s41586-019-1568-6 Medline

23. M. Guo, F. Böttcher, J. Hertkorn, J.-N. Schmidt, M. Wenzel, H. P. Büchler, T.
Langen, T. Pfau, The low-energy Goldstone mode in a trapped dipolar supersolid. Nature 574, 386-389 (2019). doi:10.1038/s41586-019-1569-5 Medline

24. G. Natale, R. M. W. van Bijnen, A. Patscheider, D. Petter, M. J. Mark, L. Chomaz, F. Ferlaino, Excitation spectrum of a trapped dipolar supersolid and its experimental evidence. Phys. Rev. Lett. 123, 050402 (2019). doi:10.1103/PhysRevLett.123.050402 Medline

25. N. L. ludice, F. Palumbo, New isovector collective modes in deformed nuclei. Phys. Rev. Lett. 41, 1532-1534 (1978). do:10.1103/PhysRevLett.41.1532

26. D. Guéry-Odelin, S. Stringari, Scissors mode and superfluidity of a trapped BoseEinstein condensed gas. Phys. Rev. Lett. 83, 4452-4455 (1999). doi:10.1103/PhysRevLett.83.4452

27. F. Zambelli, S. Stringari, Moment of inertia and quadrupole response function of a trapped superfluid. Phys. Rev. A 63, 033602 (2001). do: $10.1103 / P h y s R e v A .63 .033602$

28. O. M. Maragò, S. A. Hopkins, J. Arlt, E. Hodby, G. Hechenblaikner, C. J. Foot, Observation of the scissors mode and evidence for superfluidity of a trapped bose-einstein condensed Gas. Phys. Rev. Lett. 84, 2056-2059 (2000). doi:10.1103/PhysRevLett.84.2056 Medline

29. S. M. Roccuzzo, A. Gallemí, A. Recati, S. Stringari, Rotating a supersolid dipolar gas. Phys. Rev. Lett. 124, 045702 (2020). doن:10.1103/PhysRevL ett.124.045702 Medline

30. Materials and methods are available as supplementary materials

31. Y. Pomeau, S. Rica, Dynamics of a model of supersolid. Phys. Rev. Lett. 72, 24262429 (1994). doi:10.1103/PhysRevLett.72.2426 Medline

32. R. M. W. van Bijnen, N. G. Parker, S. J. J. M. F. Kokkelmans, A. M. Martin, D. H. J. O'Dell, Collective excitation frequencies and stationary states of trapped dipolar Bose-Einstein condensates in the Thomas-Fermi regime. Phys. Rev. A 82, 033612 (2010). doi:10.1103/PhysRevA.82.033612

33. I. Ferrier-Barbut, M. Wenzel, F. Böttcher, T. Langen, M. Isoard, S. Stringari, T. Pfau, Scissors mode of dipolar quantum droplets of dysprosium atoms. Phys. Rev. Lett. 120, 160402 (2018). doi:10.1103/PhysRevLett.120.160402 Medline

34. A. L. Fetter, Vortex nucleation in deformed rotating cylinders. J. Low Temp. Phys. 16, 533-555 (1974). doi:10.1007/BF00654901

35. A. S. C. Rittner, J. D. Reppy, Observation of classical rotational inertia and nonclassical supersolid signals in solid ${ }^{4}$ He below $250 \mathrm{mK}$. Phys. Rev. Lett. 97, 165301 (2006). doi:10.1103/PhysRevLett.97.165301Medline

36. C. Josserand, Y. Pomeau, S. Rica, Coexistence of ordinary elasticity and superfluidity in a model of a defect-free supersolid. Phys. Rev. Lett. 98, 195301 (2007). doi:10.1103/PhysRevLett 98.195301 Medline

37. A. Aftalion, X. Blanc, R. L. Jerrard, Nonclassical rotational inertia of a supersolid. Phys. Rev. Lett. 99, 135301 (2007). doi:10.1103/PhysRevLett 99.135301 Medline

38. L. Tanzi, J. G. Maloberti, G. Biagioni, A. Fioretti, C. Gabbanini, G. Modugno, Data for: Evidence of superfluidity in a dipolar supersolid from nonclassical rotational inertia. Zenodo (2020); https://doi.org/10.5281/zenodo.4288848.

39. E. Lucioni, L. Tanzi, A. Fregosi, J. Catani, S. Gozzini, M. Inguscio, A. Fioretti, C. Gabbanini, G. Modugno, Dysprosium dipolar Bose-Einstein condensate with broad Feshbach resonances. Phys. Rev. A (Coll. Park) 97, 060701 (2018) doi:10.1103/PhysRevA.97.060701

40. F. Böttcher, M. Wenzel, J. N. Schmidt, M. Guo, T. Langen, I. Ferrier-Barbut, T. Pfau, R. Bombín, J. Sánchez-Baena, J. Boronat, F. Mazzanti, Dilute dipolar quantum droplets beyond the extended Gross-Pitaevskii equation. Phys. Rev. Res. 1 , 033088 (2019). doi:10.1103/PhysRevResearch.1.033088

41. S. Stringari, Collective excitations of a trapped Bose-condensed gas. Phys. Rev. Lett. 77, 2360-2363 (1996). doi:10.1103/PhysRevLett.77.2360 Medline

42. M. Edwards, C. W. Clark, P. Pedri, L. Pitaevskii, S. Stringari, Consequence of superfluidity on the expansion of a rotating Bose-Einstein condensate. Phys. Rev. Lett. 88, 070405 (2002). doi:10.1103/PhysRevLett.88.070405 Medline

43. G. Hechenblaikner, E. Hodby, S. A. Hopkins, O. M. Maragò, C. J. Foot, Direct observation of irrotational flow and evidence of superfluidity in a rotating BoseEinstein condensate. Phys. Rev. Lett. 88, 070406 (2002). doi:10.1103/PhysRevLett 88.070406 Medline

44. M. Modugno, G. Modugno, G. Roati, C. Fort, M. Inguscio, Scissors mode of an expanding Bose-Einstein condensate. Phys. Rev. A 67, 023608 (2003). doi:10.1103/PhysRevA.67.023608

45. O. Maragò, G. Hechenblaikner, E. Hodby, C. Foot, Temperature dependence of 
damping and frequency shifts of the scissors mode of a trapped Bose-Einstein condensate. Phys. Rev. Lett. 86, 3938-3941 (2001).

doi:10.1103/PhysRevLett.86.3938 Medline

46. M. J. Wright, S. Riedl, A. Altmeyer, C. Kohstall, E. R. S. Guajardo, J. H. Denschlag, R. Grimm, Finite-temperature collective dynamics of a Fermi gas in the BEC-BCS crossover. Phys. Rev. Lett. 99, 150403 (2007). doi:10.1103/PhysRevLett.99.150403 Medline

47. S. Giorgini, Collisionless dynamics of dilute Bose gases: Role of quantum and thermal fluctuations. Phys. Rev. A 61, 063615 (2000).

doi:10.1103/PhysRevA.61.063615

\section{ACKNOWLEDGMENTS}

We thank E. Lucioni for contributions to the early stages of the experiment, A. Gallemi, A. Recati, S. Roccuzzo and S. Stringari for discussions and for providing the theoretical data, D.E. Galli for discussions, A. Barbini, F. Pardini, M. Tagliaferri and M. Voliani for technical assistance. Funding: This work received funding by the EC-H2020 research and innovation program (Grant 641122 -

QUIC). Author contributions: L.T., A.F., C.G and G.M. developed the experimental methods; L.T., J.G.M., G.B. and C.G. acquired the experimental data; L.T., J.G.M., G.B., C.G. and G.M. analyzed the data; G. B. performed the comparison with Leggett's theory; all authors contributed to the discussion and to the manuscript preparation. Competing interests: Authors declare no competing interests. Data and materials availability: All data needed to evaluate the conclusions in the paper are present in the main text or the supplementary materials. All data have been uploaded to Zenodo (38).

\section{SUPPLEMENTARY MATERIALS}

science. sciencemag.org/cgi/content/full/science.aba4309/DC1

Materials and Methods

Fig. S1

Table S1

References (39-47)

4 December 2019; accepted 2 February 2021

Published online 18 February 2021

10.1126/science.aba4309 
A

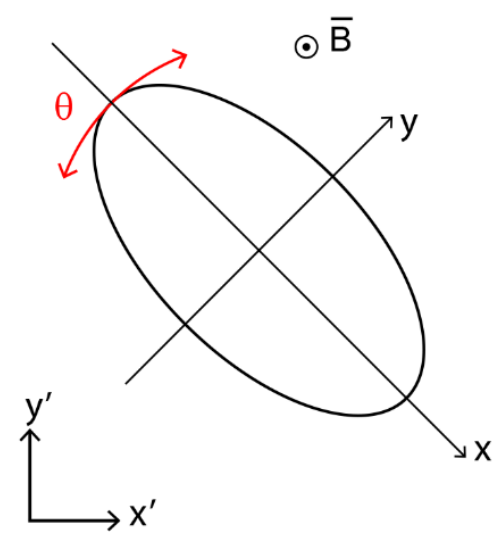

B
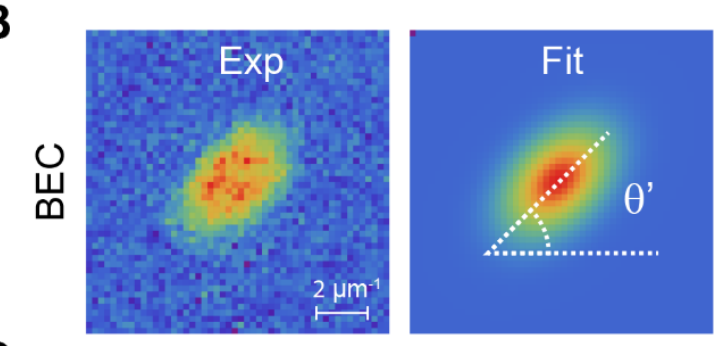

C

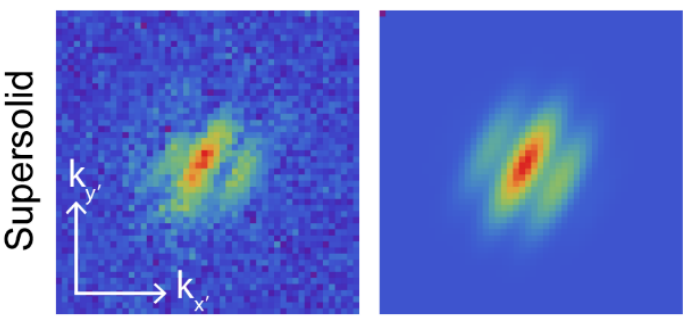

\section{D}

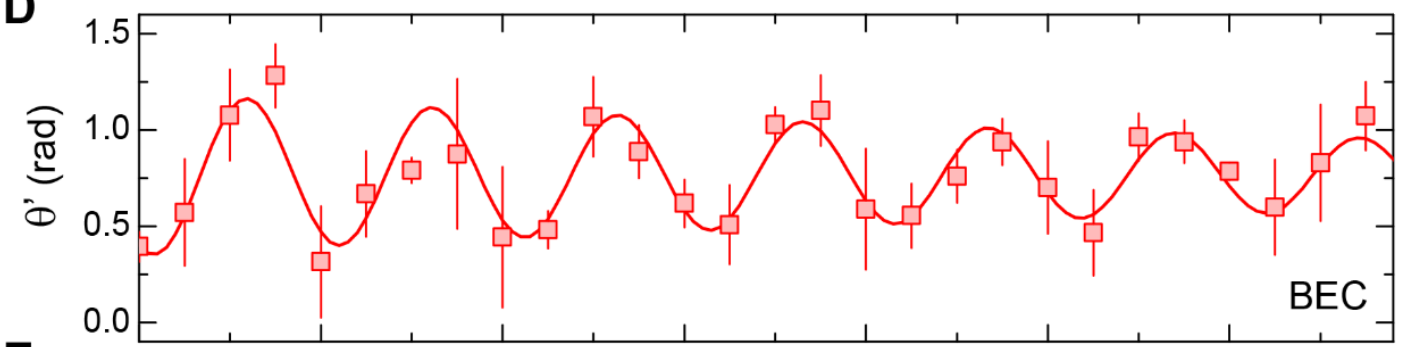

E

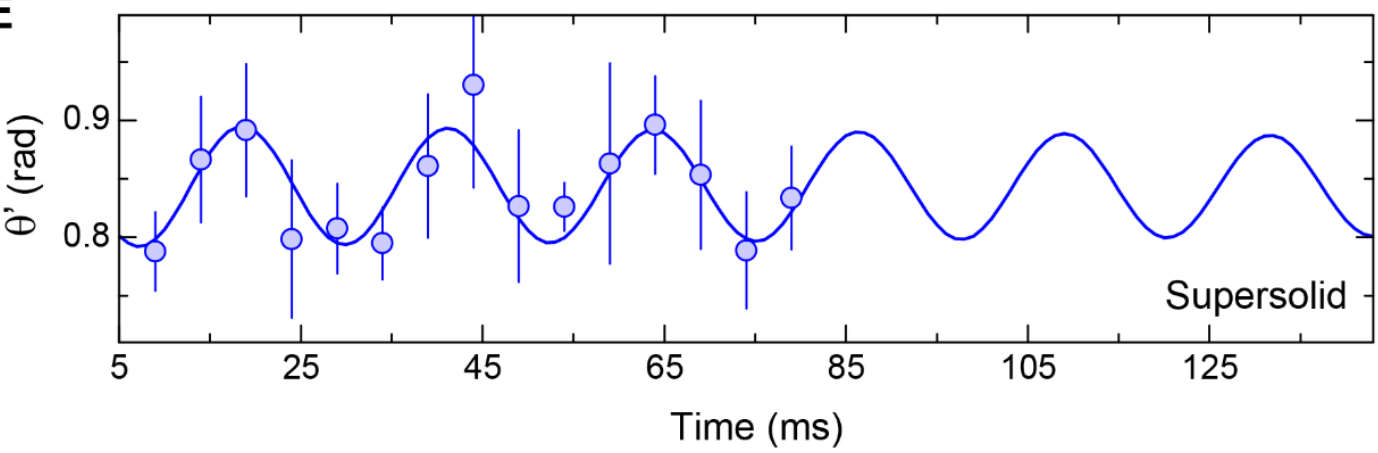

Fig. 1. Scissors mode measurements. (A) Sketch of the experimental geometry: the atomic system (ellipse) is trapped in an anisotropic potential with eigenaxes $x$ and $y$. A sudden rotation of the trapping potential excites an angular oscillation $\theta(t)$ (red arrows). ( $\mathrm{B}$ and $\mathrm{C}$ ) Examples of the experimental distributions after free expansion and of the corresponding two-dimensional fits used for extracting the oscillation angle $\theta^{\prime}$ after the free expansion in (B) BEC regime $\left(\varepsilon_{d d}=1.14\right)$; (C) Supersolid regime $\left(\varepsilon_{d d}=1.45\right)$. (D and E) Time evolution of the angle $\theta^{\prime}(t)$ : (D) BEC regime; (E) supersolid regime. Error bars represent the standard deviation of 4-8 measurements. 


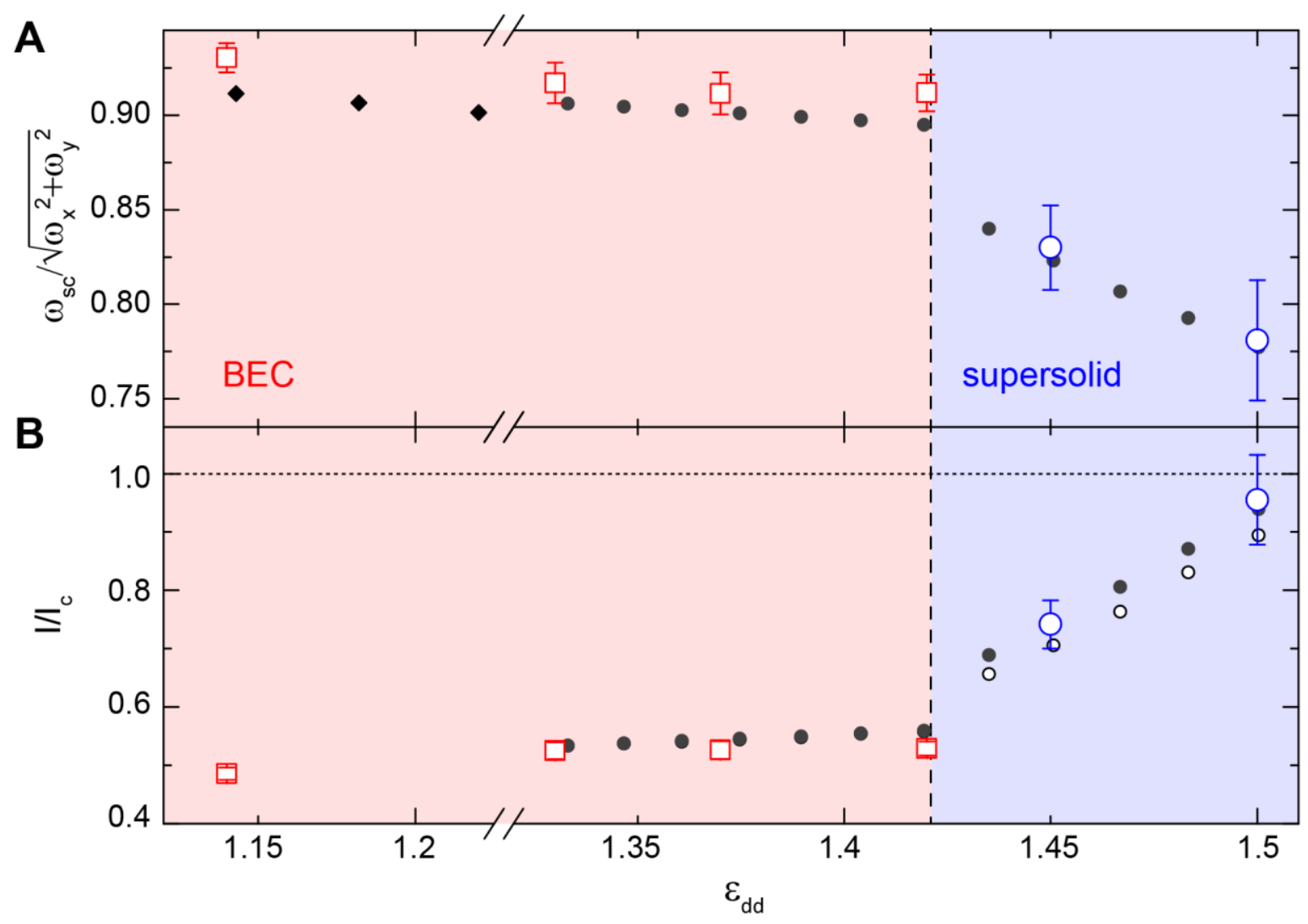

Fig. 2. Scissors mode frequency and moment of inertia vs the interaction parameter. (A) Scissors mode frequencies. Large circles and squares are the experimental measurements. Black diamonds and dots are the mean-field and beyond-mean-field theoretical predictions, respectively (29, 32). (B) Moment of inertia. Large squares and circles are derived from Eq. 1, using the experimental measurements of the scissors frequencies and the theoretical $\beta$ (29); black dots are the numerical simulation (29). Small open dots are the theoretical prediction for $\beta^{2}$ (29). Error bars are one standard deviation (30). In the experiment, $\varepsilon_{d d}$ has a calibration uncertainty of $3 \%$. The dashed line separating BEC and supersolid regimes was determined numerically (29). 


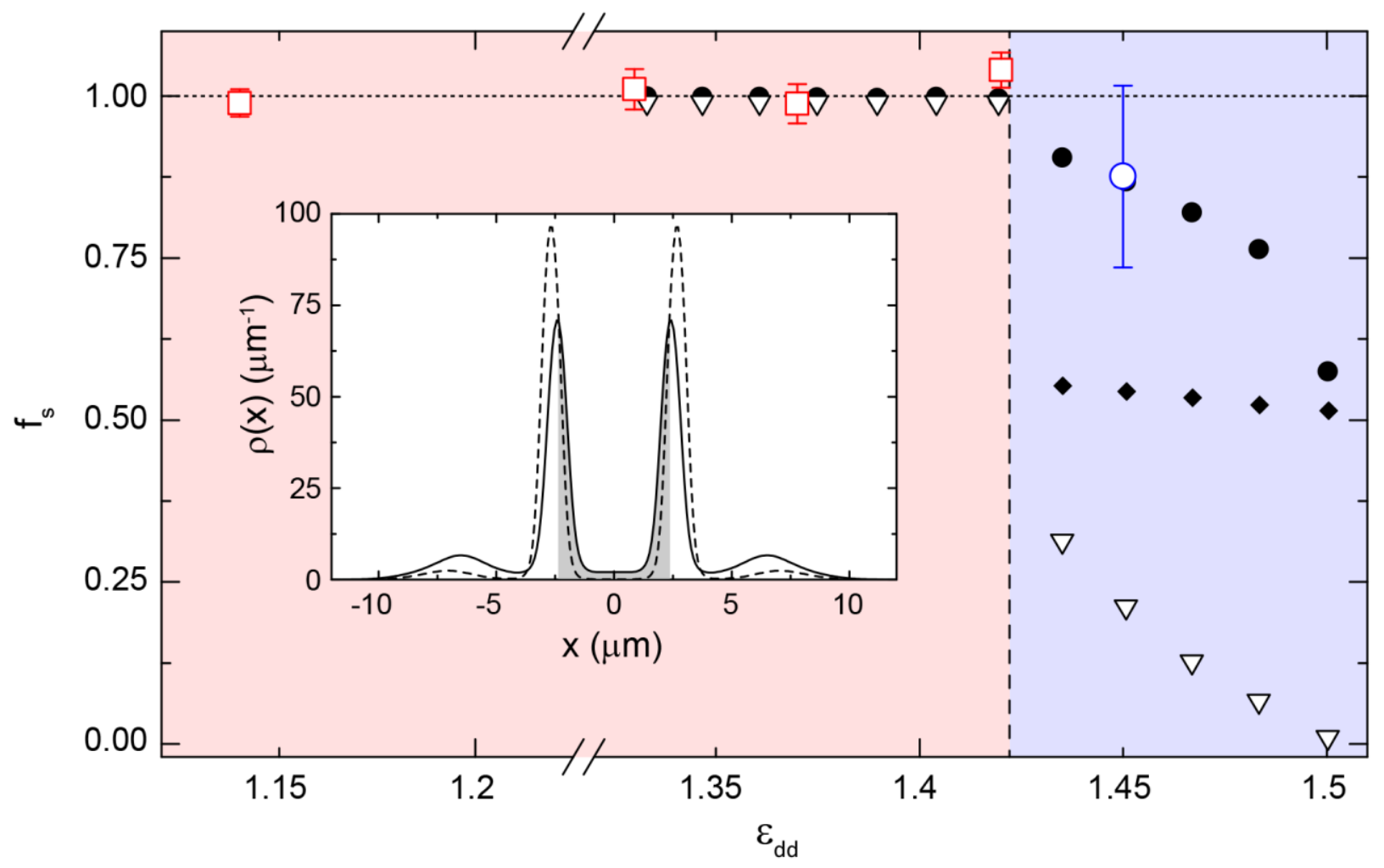

Fig. 3. Superfluid fraction from BEC to supersolid. Red squares and blue circles are the superfluid fraction from the experimentally measured scissors frequency and the theoretical $\beta$, using Eq. 3. Black dots are the superfluid fraction from the theoretical frequency (29). Open triangles are the upper limit for the one-dimensional superfluid fraction from Eq. 4. Diamonds are the estimated superfluid fraction of independent droplets. Inset: Calculated mean density distribution (30) for $\varepsilon_{d d}=1.45$ (continuous line) and $\varepsilon_{d d}=1.5$ (dashed line); the gray region is the region of integration for Eq. 4. 